\title{
Chest radiography or computed tomography for COVID-19 pneumonia? Comparative study in a simulated triage setting
}

\author{
Nicola Sverzellati ${ }^{1}$, Christopher J. Ryerson ${ }^{2}$, Gianluca Milanese $^{1}$, Elisabetta A. Renzoni ${ }^{3}$, Annalisa Volpi $^{4}$, \\ Paolo Spagnolo ${ }^{5}$, Francesco Bonella $\mathbb{1 0}^{6}$, Ivan Comelli ${ }^{7}$, Paola Affanni (i) $^{8}$, Licia Veronesi ${ }^{8}$, \\ Carmelinda Manna ${ }^{1}$, Andrea Ciuni ${ }^{1}$, Carlotta Sartorio ${ }^{1}$, Giulia Tringali ${ }^{1}$, Mario Silva ${ }^{1}$, Emanuele Michieletti ${ }^{9}$, \\ Davide Colombi ${ }^{9}$ and Athol U. Wells ${ }^{3}$
}

\begin{abstract}
${ }^{1}$ Scienze Radiologiche, Dipartimento di Medicina e Chirurgia, University Hospital of Parma, Parma, Italy. ${ }^{2}$ Dept of Medicine, University of British Columbia and Centre for Heart Lung Innovation, St Paul's Hospital, Vancouver, BC, Canada. ${ }^{3}$ Interstitial Lung Disease Unit, Royal Brompton Hospital, Imperial College London, London, UK. ${ }^{4} 1$ st Anesthesia and Intensive Care Unit, University Hospital of Parma, Parma, Italy. ${ }^{5}$ Respiratory Disease Unit, Dept of Cardiac, Thoracic, Vascular Sciences and Public Health, University of Padova, Padua, Italy. ${ }^{6}$ Center for Interstitial and Rare Lung Diseases, Pneumology Dept, Ruhrandklinik University Hospital, University of Duiburg-Essen, Essen, Germany. ${ }^{7}$ Unità Operativa Pronto Soccorso e Medicina d'Urgenza, Azienda Ospedaliero-Universitaria di Parma, Parma, Italy. ${ }^{8}$ Dept of Medicine and Surgery, University of Parma, Parma, Italy. ${ }^{9}$ Dept of Radiological Functions, Radiology Unit, "Guglielmo da Saliceto" Hospital, Piacenza, Italy.
\end{abstract}

Corresponding author: Nicola Sverzellati (nicola.sverzellati@unipr.it)

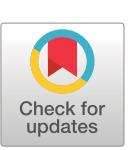

This version is distributed under the terms of the Creative Commons Attribution Non-Commercial Licence 4.0. For commercial reproduction rights and permissions contact permissions@ersnet.org

This article has supplementary material available from erj.ersjournals.com

Received: 2 Nov 2020 Accepted: 27 Jan 2021

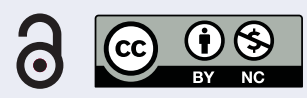

Shareable abstract (@ERSpublications)

This study does not support the routine use of CT to stage disease extent in COVID-19 pneumonia, despite superior interobserver agreement, as chest radiography extent is an equally powerful prognostic determinant https://bit.ly/3rdJuxj

Cite this article as: Sverzellati N, Ryerson CJ, Milanese G, et al. Chest radiography or computed tomography for COVID-19 pneumonia? Comparative study in a simulated triage setting. Eur Respir J 2021; 58: 2004188 [DOI: 10.1183/13993003.04188-2020].

\section{Abstract}

Introduction For the management of patients referred to respiratory triage during the early stages of the severe acute respiratory syndrome coronavirus type 2 (SARS-CoV-2) pandemic, either chest radiography or computed tomography (CT) were used as first-line diagnostic tools. The aim of this study was to compare the impact on the triage, diagnosis and prognosis of patients with suspected COVID-19 when clinical decisions are derived from reconstructed chest radiography or from CT.

Methods We reconstructed chest radiographs from high-resolution CT (HRCT) scans. Five clinical observers independently reviewed clinical charts of 300 subjects with suspected COVID-19 pneumonia, integrated with either a reconstructed chest radiography or HRCT report in two consecutive blinded and randomised sessions: clinical decisions were recorded for each session. Sensitivity, specificity, positive predictive value (PPV), negative predictive value (NPV) and prognostic value were compared between reconstructed chest radiography and HRCT. The best radiological integration was also examined to develop an optimised respiratory triage algorithm.

Results Interobserver agreement was fair (Kendall's $W=0.365, \mathrm{p}<0.001$ ) by the reconstructed chest radiography-based protocol and good (Kendall's $W=0.654, \mathrm{p}<0.001$ ) by the CT-based protocol. NPV assisted by reconstructed chest radiography (31.4\%) was lower than that of HRCT (77.9\%). In case of indeterminate or typical radiological appearance for COVID-19 pneumonia, extent of disease on reconstructed chest radiography or HRCT were the only two imaging variables that were similarly linked to mortality by adjusted multivariable models

Conclusions The present findings suggest that clinical triage is safely assisted by chest radiography. An integrated algorithm using first-line chest radiography and contingent use of HRCT can help optimise management and prognostication of COVID-19.

\section{Introduction}

Despite worldwide efforts to halt its transmission, severe acute respiratory syndrome coronavirus type 2 (SARS-CoV-2) has affected more than 30 million individuals and caused nearly 1 million deaths as of late 
September 2020 [1, 2]. After the initial outbreak, most countries have prepared their healthcare systems to face the pandemic. Although highly desirable, global and shared preparedness planning has faced political, institutional, social, environmental and technological challenges [3, 4]. A recent international survey reported substantial heterogeneity in the diagnostic approach to COVID-19 pneumonia within and among countries and continents [4].

To date, molecular testing is used in both symptomatic or asymptomatic subjects with risk of contamination. However, the use of imaging, particularly the choice of imaging technique, is still a matter of debate [5-8]. Molecular and imaging testing are helpful in different aspects of the disease, and their integration should be driven by dynamic protocols to be adapted as knowledge of the disease improves. The use of real-time reverse transcription-PCR (RT-PCR) was adapted to the massive demands by shortening the reaction time (and thus the reporting time), yet it is still challenged by a substantial proportion of false negatives [9]. Conversely, imaging can show signs of pneumonia in patients with negative RT-PCR but clinically suspected COVID-19, thus offering a potential role in supporting rapid decision making $[9,10]$. The use of imaging was thus recommended for patients who present at triage with moderate-to-severe features of COVID-19 pneumonia regardless of RT-PCR results [7].

Different imaging approaches are available and have been discussed in the recent COVID-19 literature, including chest radiography and computed tomography (CT). There is still no international consensus on the integrated use of chest radiography or CT for clinical assessment and management of subjects with suspected COVID-19 pneumonia [4-6, 11-17]. Most concerns are focused on the accuracy of these tests, individual resources, and risk of infection for radiographers and other healthcare employees. However, the scientific debate lacks important evidence on the impact of chest radiography and CT on triage decisions and patient care.

In this study, we sought to use a post-processing imaging technique to retrospectively reconstruct chest radiographs from CT scans, and compare the impact of these two imaging tools on the initial clinical triage, diagnosis and prognosis of patients with suspected COVID-19.

\section{Methods}

\section{Study population}

The study population comprised patients who had been evaluated with chest high-resolution CT (HRCT) scan by COVID-19 respiratory triage at the University Hospital of Parma (Parma, Italy), which is located in one of the most affected areas in Northern Italy. In brief, patients were screened for symptoms (e.g. fever and dyspnoea) and oxygen saturation. Patients with moderate-to-severe pulmonary involvement (e.g. oxygen saturation $\leqslant 95 \%$ ) underwent HRCT. Given the turnaround times for SARS-CoV-2 testing results (e.g. ranging from 2 to $>48 \mathrm{~h}$ ), a presumptive diagnosis based on clinico-radiological findings was considered for swift decision making, such as discharge, recommendation for home quarantine or hospitalisation in different hospital areas, including either dedicated COVID-19 pavilions or non-COVID-19 wards. Details on clinical triage were previously reported [8].

The study derivation cohort was built up by including 300 patients who consecutively underwent HRCT at the Parma triage from 29 February 2020 to 7 March 2020, as follows: the first 200 patients consecutively admitted to the triage with moderate-to-severe respiratory clinical impairment were combined with the first 100 patients consecutively admitted with mild respiratory clinical impairment (e.g. oxygen saturation 96$98 \%$, hyperthermia or tachypnoea) (figure 1). The addition of this subgroup ensured that the full spectrum of COVID-19 severity was evaluated.

The study results were externally validated in a cohort of 104 patients (validation cohort) consecutively evaluated at a neighbouring hospital during the same time frame ("Guglielmo da Saliceto" Hospital, Piacenza, Italy), which adopted a similar diagnostic protocol (supplementary material).

This retrospective study was approved by the referring local review board. Informed consent was obtained from the study patients.

\section{Imaging technique}

Details on CT scanners and the HRCT technique are reported in the supplementary material. HRCT scans allow for various post-processing reconstruction algorithms, including average intensity projection (AIP) [18]. AIP images represent the average of each component attenuation value encountered by the X-ray beam through an object. By manipulating the slab thickness of the coronal AIP mages, it was possible to obtain images analogous to frontal chest radiography, hereafter called reconstructed chest radiographs (see the 


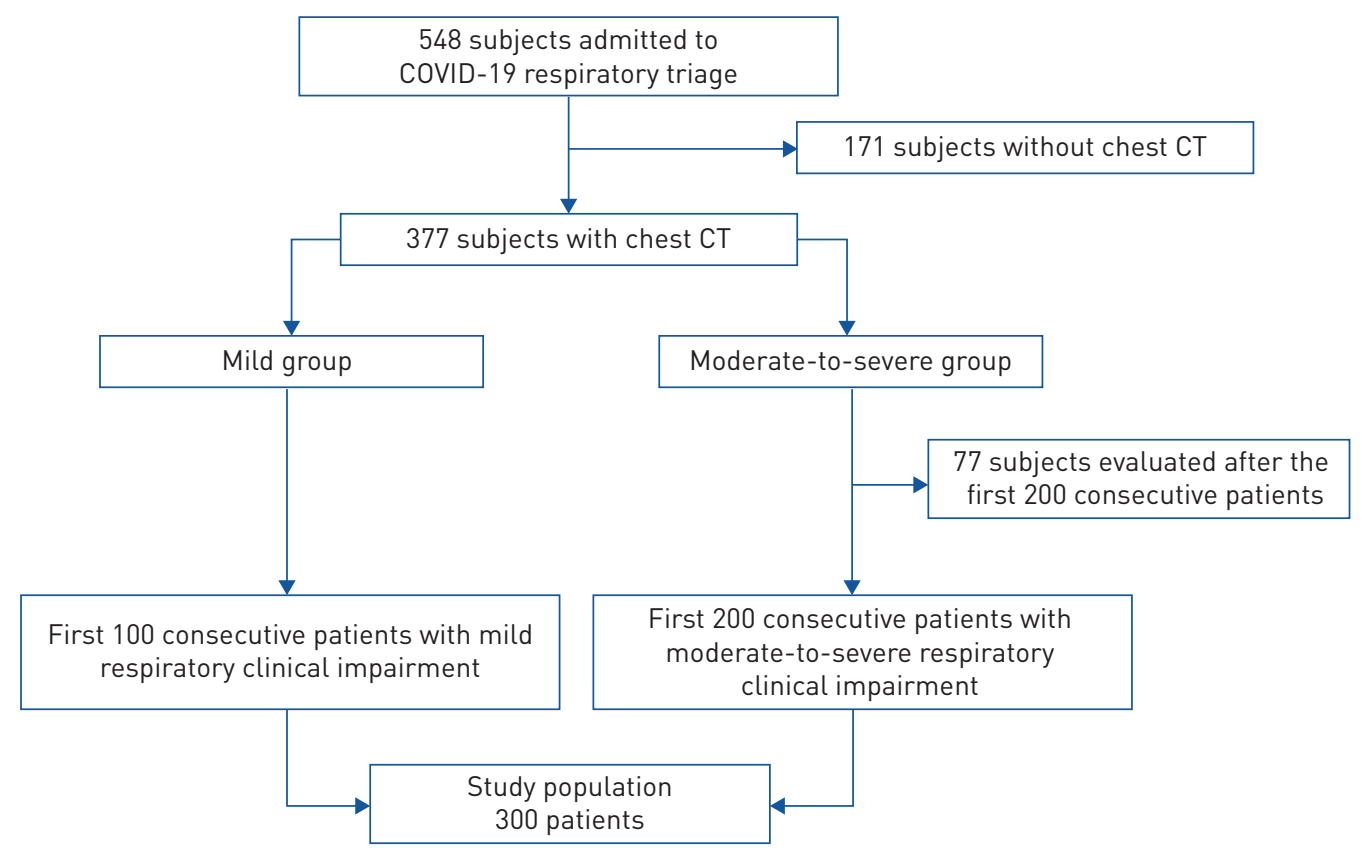

FIGURE 1 Diagram illustrating the selection of the derivation study cohort. CT: computed tomography.

videoclip in the supplementary material and figures 2-4). Further illustrations and technical details of the conversion from HRCT to reconstructed chest radiography imaging as well as evaluation of reconstructed chest radiography consistency with standard chest radiography are reported in the supplementary material.

HRCTs were prospectively scored by a senior chest radiologist (N.S.; 16 years of experience in imaging of interstitial lung disease). He recorded individual HRCT abnormalities and graded the HRCTs into four diagnostic categories: normal, alternative diagnosis (to be specified), indeterminate or typical for COVID-19 pneumonia [8]. The total extent of pulmonary disease was scored to the nearest $5 \%$.
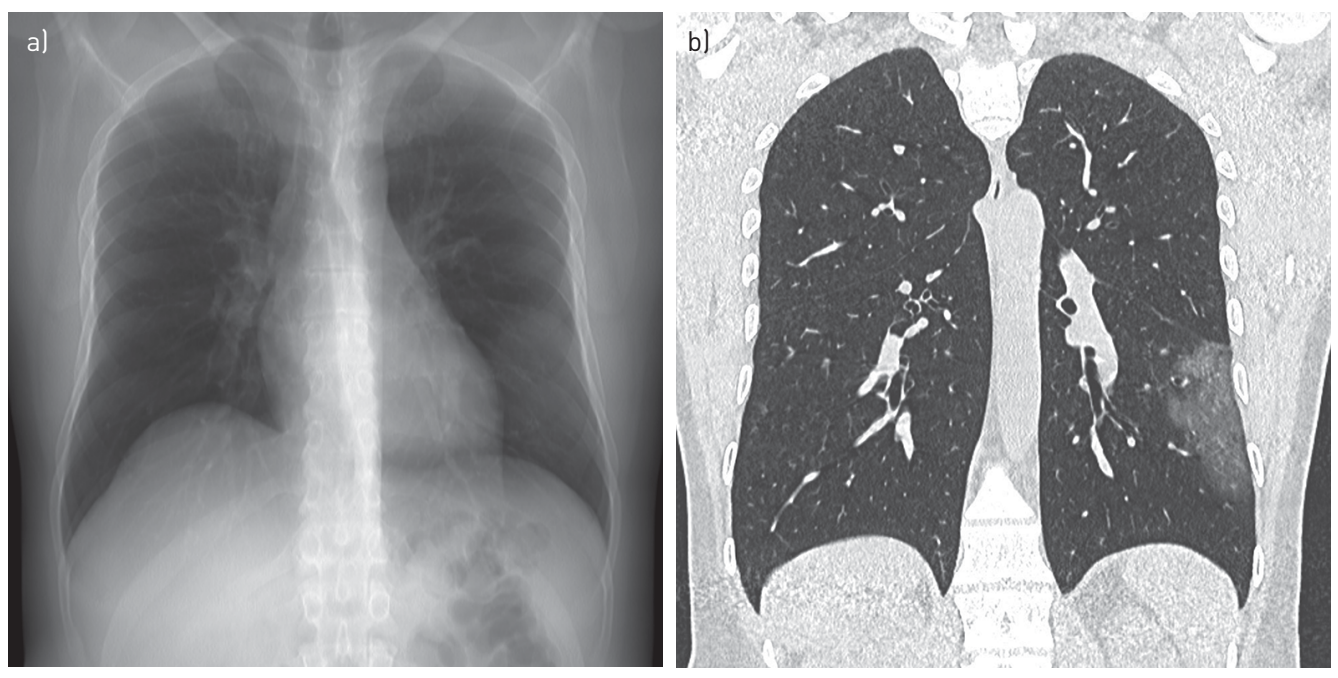

FIGURE 2 a) Reconstructed chest radiography and b) coronal multiplanar reconstruction of high-resolution computed tomography (HRCT) of a 41-year-old male patient with unilateral COVID-19 pneumonia. The focal, ovoid area of ground-glass opacity in the left lower lobe was identified at both reconstructed chest radiography and HRCT by the study observers. The extent of disease at reconstructed chest radiography was scored as category $0(<20 \%)$ by both of the study observers when using the prognostic scoring system. 

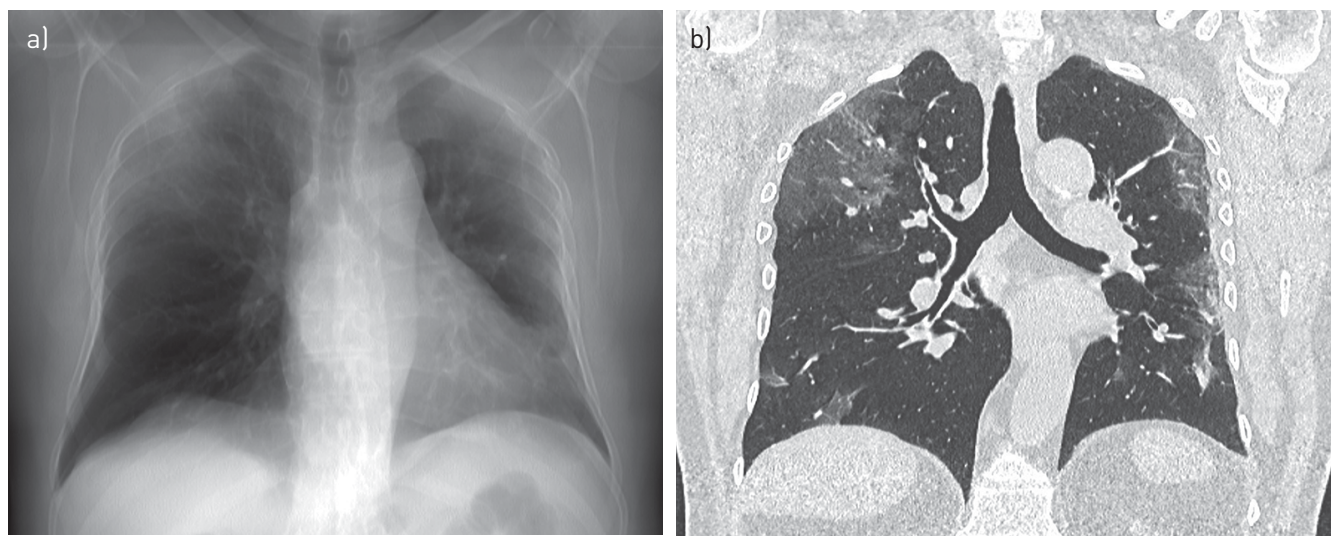

FIGURE 3 a) Reconstructed chest radiography and b) coronal multiplanar reconstruction of high-resolution computed tomography (HRCT) of a 71-year-old male patient with bilateral COVID-19 pneumonia. Bilateral, peripheral ground-glass opacities were reported at both reconstructed chest radiography and HRCT. However, disease extent was scored as category $0(<20 \%)$ on reconstructed chest radiography and category $1(20-50 \%)$ on HRCT.

In keeping with the visual scoring of the HRCT, two radiologist observers (C.M. and A.C.; 11 and 4 years of experience in chest imaging, respectively) recorded individual reconstructed chest radiography abnormalities and graded the reconstructed chest radiographs as: normal, alternative diagnosis (to be specified), indeterminate or typical for COVID-19 pneumonia. The total extent of pulmonary disease on reconstructed chest radiography was evaluated through an overall visual impression, using a four-point scale: $0=$ no parenchymal abnormality, $1=$ extent $<20 \%$, $2=$ extent $20-50 \%$ and $3=$ extent $>50 \%$. Interobserver discrepancies for both diagnostic categories and disease extent were resolved by consensus.

\section{Clinical assessment in a triage-like setting}

Clinical data for each patient were jointly reported in data sheets by a consultant anaesthesiologist (A.V.) and a radiologist (G.M.) (table 1). These data were assembled into individual clinical charts that were given for simulation of clinical management to each clinical observer of this study. RT-PCR test results were not included in the clinical charts in order to simulate an environment where turnaround times were long.

Five clinical observers from three different countries participated in the study. These comprised three physicians (A.V., I.C. and F.B.) who worked at a HRCT-based triage and two physicians (P.S. and E.A.R.)
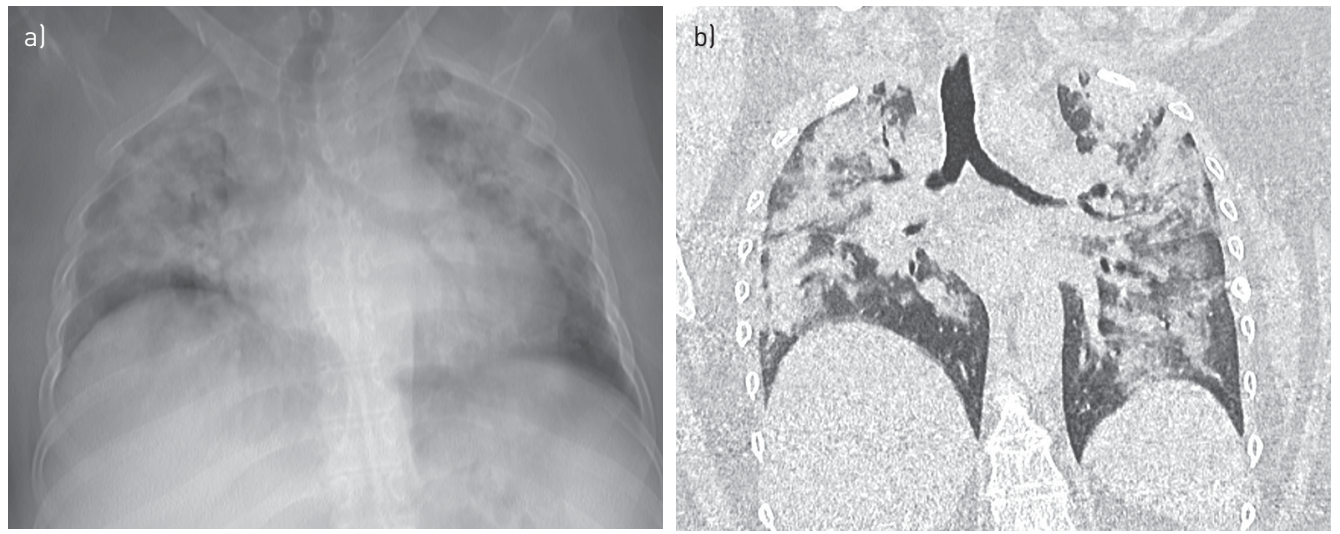

FIGURE 4 a) Reconstructed chest radiography and b) coronal multiplanar reconstruction of high-resolution computed tomography (HRCT) of a 41-year-old female patient with severe COVID-19 pneumonia. Bilateral, diffuse ground-glass opacities and consolidations occupy most of the lung parenchyma, and the disease extent was scored as category $2(>50 \%)$ both on reconstructed chest radiography and HRCT. 
TABLE 1 Baseline characteristics, presentation vitals and laboratory results of patients admitted to the respiratory triage for COVID-19 for the derivation and validation cohorts

\begin{tabular}{|c|c|c|}
\hline & Derivation cohort & Validation cohort \\
\hline Patients & 300 & 104 \\
\hline Age years & $66.8 \pm 15.8$ & $70.1 \pm 14.3$ \\
\hline \multicolumn{3}{|l|}{ Sex } \\
\hline Male & $188(62.7)$ & $71(68.3)$ \\
\hline Female & $112(37.3)$ & $33(31.7)$ \\
\hline \multicolumn{3}{|c|}{$\begin{array}{l}\text { Contact with suspected or confirmed COVID-19 } \\
\text { cases }\end{array}$} \\
\hline Yes & $72(24.0)$ & $23(22.2)$ \\
\hline No & $49(16.3)$ & $77(74.0)$ \\
\hline Not reported & $179(59.7)$ & $4(3.8)$ \\
\hline History of fever & $231(77.0)$ & $92(88.5)$ \\
\hline Duration of fever days & $6(3-7)$ & $5.5(2-7.5)$ \\
\hline Temperature ${ }^{\circ} \mathrm{C}$ & $37(36.5-37.8)$ & $37.3(36.5-38.2)$ \\
\hline Temperature $\geqslant 37.5^{\circ} \mathrm{C}$ & $90(30.0)$ & $44(46.3)$ \\
\hline History of cough & $171(57.0)$ & $42(40.0)$ \\
\hline Duration of cough days & $5(3-7)$ & $3(0-7)$ \\
\hline History of dyspnoea & $109(36.3)$ & $63(60.6)$ \\
\hline Duration of dyspnoea days & $3(3-7)$ & $1(0-4)$ \\
\hline Respiratory rate $\geqslant 25$ breaths $\cdot \mathrm{min}^{-1}$ & $74(24.7)$ & $16(22.9)$ \\
\hline Oxygen saturation $\%$ & $94(90-96)$ & $92(88-95.2)$ \\
\hline Oxygen saturation $\leqslant 95 \%$ & $200(66.7)$ & $73(75.3)$ \\
\hline Hearth rate $>100$ beats $\cdot \mathrm{min}^{-1}$ & $66(22.0)$ & $15(18.5)$ \\
\hline Other symptoms & $92(30.7)$ & $22(21.2)$ \\
\hline Hypertension & $114(38.0)$ & $58(55.8)$ \\
\hline Diabetes & $42(14.0)$ & $18(17.3)$ \\
\hline Obesity & $18(6.0)$ & $9(8.7)$ \\
\hline \multicolumn{3}{|l|}{ Other comorbidities } \\
\hline Cancer & $33(11.0)$ & $19(18.3)$ \\
\hline Chronic respiratory disease & $44(14.7)$ & $15(14.4)$ \\
\hline Kidney disease & $12(4.0)$ & $13(12.5)$ \\
\hline Liver disease & $1(0.3)$ & $2(1.9)$ \\
\hline No other comorbidities reported & $210(70.0)$ & $55(52.9)$ \\
\hline \multicolumn{3}{|l|}{ Laboratory measures" } \\
\hline White blood cell count $\times 10^{9} \mathrm{~L}^{-1}$ & $6.4(4.6-8.7)$ & $7.4(5.1-9.5)$ \\
\hline Sodium $\mathrm{mmol} \cdot \mathrm{L}^{-1}$ & $137(135-139)$ & $136(133-139)$ \\
\hline Aspartate aminotransferase $\mathrm{U} \cdot \mathrm{L}^{-1}$ & $42(32-62)$ & $44(30-63)$ \\
\hline Alanine aminotransferase $\mathrm{U} \cdot \mathrm{L}^{-1}$ & $28(19-42)$ & $28(20-49)$ \\
\hline C-reactive protein $\mathrm{mg} \cdot \mathrm{L}^{-1}$ & $83(27-148)$ & $82(36-150)$ \\
\hline \multicolumn{3}{|c|}{ Reconstructed chest radiography categories } \\
\hline Normal & 85 & 18 \\
\hline Alternative diagnosis & 7 & 0 \\
\hline Indeterminate & 40 (median extent: $\leqslant 20 \%$ ) & 16 (median extent: $\leqslant 20 \%$ ) \\
\hline Typical for COVID-19 pneumonia & $\begin{array}{l}168 \text { (median } \\
\text { extent: } 21-50 \%)\end{array}$ & $\begin{array}{l}70 \text { (median } \\
\text { extent: } 20-50 \% \text { ) }\end{array}$ \\
\hline \multicolumn{3}{|l|}{ HRCT categories } \\
\hline Normal & 40 & 6 \\
\hline Alternative diagnosis & 35 (mean extent: 5.6\%) & 1 \\
\hline Indeterminate & 41 (mean extent: $28.4 \%$ ) & 13 (mean extent: $32.7 \%$ ) \\
\hline Typical for COVID-19 pneumonia & 184 (mean extent: 35\%) & 84 (mean extent: $36.5 \%)$ \\
\hline
\end{tabular}

whose hospital protocol proposed a chest radiography-based triage. Details on the study observer characteristics are given in the supplementary material. Each clinical observer was asked to independently review the full set of clinical charts twice according to two different settings.

The first review was designed to simulate a reconstructed chest radiography-based integrated clinico-radiological protocol: each clinical observer reviewed the data sheet and reconstructed chest 
radiography report for each patient. Each observer was asked to provide a clinical decision according to one of the following options: discharge, hospitalisation in non-COVID-19 area, home quarantine, hospitalisation in COVID-19 area or further work-up by chest HRCT.

The second review was designed to simulate a HRCT-based integrated clinico-radiological protocol: 2 days after the first review was completed, each clinical observer started the second review of the data sheet and HRCT report for each patient. Each observer was asked to provide a clinical decision according to one of the following options: discharge, hospitalisation in non-COVID-19 area, home quarantine or hospitalisation in COVID-19 area.

The study observers were informed that the triage setting was supposed to simulate a pandemic environment with a high influx of subjects with suspected COVID-19 pneumonia and that the clinical decision could be expressed in the absence of any resource constraints.

\section{Statistical analysis}

The study analysis compared the frequency of each clinical decision category and its consistency between the "reconstructed chest radiography-based protocol" and the "HRCT-based protocol", by means of both intra- and interobserver analysis. Either the Chi-squared or McNemar test was used to compare reconstructed chest radiography and HRCT diagnostic categories and RT-PCR results. Radiological data were compared between reconstructed chest radiography and HRCT by the weighted $\kappa$ coefficient $\left(\kappa_{\mathrm{w}}\right)$ to evaluate interobserver agreement. The Kendall $W$-test was used to evaluate the overall interobserver clinical decision agreement by the reconstructed chest radiography-based or HRCT-based protocol. Further details are provided in the supplementary material.

Spearman's rank correlation coefficient was used to evaluate the correlation between reconstructed chest radiography extent and HRCT extent of disease.

Sensitivity, specificity, positive predictive value (PPV) and negative predictive value (NPV) of both reconstructed chest radiography and HRCT diagnostic categories were calculated against RT-PCR by grouping normal to alternative diagnosis and indeterminate to typical for COVID-19 pneumonia diagnostic categories, respectively.

Unadjusted and multivariable logistic regression analyses were used to identify the contribution of clinical and radiological variables to mortality prediction. Multivariable models included age, sex, duration of symptoms at triage and a comorbidity score of $0-4$, obtained by summing the presence ( 1 point for each) of individual comorbidities consistently associated with a poor outcome in previous reports (diabetes, hypertension, cardiovascular disease and obesity). The classification performance of the models was evaluated by the area under the receiver operating characteristic curve (AUC).

Based on these models, we constructed a user-friendly five-point scale for both CT and chest radiography, integrating age and extent of disease on imaging (the only two variables emerging as strong independent determinants of mortality). These scales were externally tested in the validation cohort.

p-values $<0.05$ were considered to indicate statistically significant differences. Analyses were performed using Stata version 14 (Computing Resource Center, Santa Monica, CA, USA).

Results

\section{Study population}

The derivation cohort included 300 patients (188 males and 112 females; mean \pm sD age $66.8 \pm 15.8$ years, range 23.1-97.6 years). Clinical and laboratory characteristics are summarised in table 1 and in the supplementary material. The mean \pm SD time interval between the onset of symptoms and the triage assessment (and also HRCT scanning) was 6.3 \pm 4.4 days. A total of 248 patients underwent RT-PCR testing. A total of 162 out of 248 (65.3\%) subjects tested positive at initial RT-PCR. Repeat RT-PCR testing was performed if the first RT-PCR was negative, identifying an additional 28 positive cases, thus resulting in an overall 190 out of 248 (76.6\%) cases positive by RT-PCR. Vital status was ascertained for 295 out of 300 (98.3\%) subjects. All patients were followed until death or a minimum of 30 days (range of follow-up 1-153 days).

The characteristics of the derivation and validation cohorts are summarised in table 1. 
TABLE 2 Management according to the reconstructed chest radiography-based protocol and the high-resolution computed tomography (HRCT)-based protocol

\begin{tabular}{lccccc} 
& \multicolumn{5}{c}{ Observer } \\
\cline { 2 - 6 } & $\mathbf{1}$ & $\mathbf{2}$ & $\mathbf{3}$ & $\mathbf{4}$ & $\mathbf{5}$ \\
\hline Cases & 300 & 300 & 300 & 300 & 300 \\
$\begin{array}{l}\text { Reconstructed chest radiography } \\
\quad \text { Discharge }\end{array}$ & $11(4)$ & $24(8)$ & $13(4)$ & $4(1)$ & $48(16)$ \\
$\quad$ Non-COVID-19 hospitalisation & $10(3)$ & $23(8)$ & $27(9)$ & $18(6)$ & $49(16)$ \\
$\quad$ Home quarantine & $111(37)$ & $27(9)$ & $99(33)$ & $47(16)$ & $18(6)$ \\
$\quad$ COVID-19 hospitalisation & $103(34)$ & $153(51)$ & $137(46)$ & $164(55)$ & $47(16)$ \\
$\quad$ Need for chest CT & $65(22)$ & $73(24)$ & $24(8)$ & $67(22)$ & $138(46)$ \\
HRCT & $5(2)$ & $17(6)$ & $4(1)$ & $8(3)$ & $25(8)$ \\
$\quad$ Discharge & $10(3)$ & $46(15)$ & $64(21)$ & $53(18)$ & $76(25)$ \\
$\quad$ Non-COVID-19 hospitalisation & $119(40)$ & $30(10)$ & $101(34)$ & $41(13)$ & $29(10)$ \\
$\quad$ Home quarantine & $166(55)$ & $207(69)$ & $131(44)$ & $198(66)$ & $170(57)$ \\
\hline COVID-19 hospitalisation & & & & & \\
\hline Data are presented as n or $\mathrm{n}(\%)$. & & & & \\
\hline
\end{tabular}

Inter- and intra-observer agreement for the clinical decision

The clinical decision according to either the reconstructed chest radiography-based protocol or the HRCT-based protocol is summarised in tables 2 and 3. The overall interobserver agreement by the reconstructed chest radiography-based protocol was fair (Kendall's $W=0.365, \mathrm{p}<0.001$ ) and it improved to good by the HRCT-based protocol (Kendall's $W=0.654, \mathrm{p}<0.001$ ). This was true even after stratification for categories of oxygen saturation levels (supplementary table S1). Of note, opposite trends in the agreement across the range of pre-specified oxygen saturation levels were observed: the lower the oxygen saturation, the worse the agreement by the reconstructed chest radiography-based protocol and the better the agreement by the HRCT-based protocol (supplementary table S1).

Paired interobserver agreement ranged from poor $\left(\kappa_{\mathrm{w}}=0.17\right.$; observer 1 versus 5$)$ to moderate $\left(\kappa_{\mathrm{w}}=0.51\right.$; observer 2 versus 4) by the reconstructed chest radiography-based protocol and from fair $\left(\kappa_{\mathrm{w}}=0.23\right.$; observer 1 versus 5 ) to good ( $\kappa_{\mathrm{w}}=0.75$; observer 2 versus 4 ) by the HRCT-based protocol (supplementary table S2).

Intra-observer agreement on the clinical decision by either reconstructed chest radiography or HRCT ranged from fair $\left(\kappa_{\mathrm{w}}=0.37\right)$ to good $\left(\kappa_{\mathrm{w}}=0.71\right)$ (supplementary table S3).

In the reconstructed chest radiography round, further work-up by HRCT was requested in 8-46\% of patients by the study observers. Once the HRCT scan was provided, the most frequent decision in this group was COVID-19 hospitalisation (41.7-73.2\%) (table 3). In particular, HRCT was requested by at least one observer for a total of 224 (74.7\%) patients, while a management decision without a request for HRCT (only clinico-reconstructed chest radiography findings) was expressed by all observers in 76 (25.3\%) patients (supplementary table S4). In the latter subgroup, the interobserver agreement was similar between

TABLE 3 High-resolution computed tomography (HRCT)-based recommendation for subjects for whom HRCT was requested at the reconstructed chest radiography-based round

\begin{tabular}{lccccc} 
& \multicolumn{5}{c}{ Observer } \\
\cline { 2 - 6 } & $\mathbf{1}$ & $\mathbf{2}$ & $\mathbf{3}$ & $\mathbf{4}$ & $\mathbf{5}$ \\
\hline Cases & 65 & 73 & 24 & 67 & 138 \\
Discharge & $0(0)$ & $3(4)$ & $0(0)$ & $2(3)$ & $2(1)$ \\
Non-COVID-19 hospitalisation & $2(3)$ & $15(21)$ & $8(33)$ & $22(33)$ & $27(20)$ \\
Home quarantine & $19(29)$ & $9(12)$ & $6(25)$ & $10(15)$ & $8(6)$ \\
COVID-19 hospitalisation & $44(68)$ & $46(63)$ & $10(42)$ & $33(49)$ & $101(73)$ \\
\hline Data are presented as $\mathrm{n}$ or $\mathrm{n}(\%)$. & & & & \\
\hline
\end{tabular}


reconstructed chest radiography (Kendall's $W=0.763, \mathrm{p}<0.001$ ) and HRCT (Kendall's $W=0.725, \mathrm{p}<0.001$ ). When reconstructed chest radiography was considered sufficient for decision making by all the observers, patients had higher oxygen saturation and more frequently reported a history of social contact with COVID-19-infected individuals compared with patients for whom HRCT was requested by any observer (supplementary table S4).

Reconstructed chest radiography versus $H R C T$

Reconstructed chest radiography and HRCT data are summarised in table 1.

Interobserver analysis data is provided in the supplementary material. 85 reconstructed chest radiographs were classified as normal, with 49 out of 85 (57.6\%) being false negative compared with HRCT scans, notably 30 out of 85 (35.3\%) showing HRCT abnormalities consistent with COVID-19 pneumonia (ground-glass opacity in 26 out of 30 (86.7\%)) with a mean disease extent on HRCT of 23\% (range 5$65 \%$ ). Conversely, indeterminate reconstructed chest radiography findings or reconstructed chest radiography suggestive of a non-COVID-19 disease were each reported in four out of 40 (10\%) patients with normal HRCT scans, thus representing false-positive reconstructed chest radiographs.

Of 190 patients with positive RT-PCR results, 156 (82.1\%) had positive HRCT scans and 133 (70\%) had positive reconstructed chest radiographs. Of 58 subjects with negative RT-PCR results, 28 (48.3\%) had positive HRCT scans and 31 (53.4\%) had positive reconstructed chest radiographs. The sensitivity, specificity, PPV and NPV of HRCT in indicating COVID-19 infection were 95.2\% (95\% CI 91.2-97.8\%), 32.8\% (95\% CI 21-46.3\%), 82.2\% (95\% CI 79.4-84.7\%) and 67.9\% (95\% CI 50.3-81.5\%), respectively. The sensitivity, specificity, PPV and NPV of reconstructed chest radiography in indicating COVID-19 infection were 81.6\% (95\% CI 75.3-86.8\%), 27.6\% (95\% CI 16.7-40.9\%), 78.1\% (95\% CI 75.0-80.9\%) and $31.4 \%$ (95\% CI 21.5-43.3\%), respectively.

Unadjusted associations between mortality and core clinical and imaging variables in patients with reconstructed chest radiography findings compatible with COVID-19 pneumonia are summarised in table 4. Two models examined mortality against the extent of disease on either reconstructed chest radiographs or HRCT scans, using the same four-point categorical grading system (table 4). In the logistic regression model with reconstructed chest radiography, mortality was associated with extent of disease (OR 2.38, 95\% CI 1.61-3.50; $\mathrm{p}<0.001)$. In the logistic regression model with HRCT, mortality was associated with extent of disease (OR 2.62, 95\% CI 1.67-4.10; p<0.001). Both reconstructed chest radiography and HRCT associations were robust with adjustment for age (also strongly linked to mortality), sex, duration of symptoms and comorbidity score (supplementary table S5).

A further third model was built to explore a two-point grading system for risk stratification: the extent of disease was classified by contingent categories as either limited (reconstructed chest radiography extent $<20 \%$; or reconstructed chest radiography $20-50 \%$ with HRCT extent $0-50 \%$ ) or extensive (reconstructed chest radiography extent $>50 \%$; or reconstructed chest radiography $20-50 \%$ with HRCT extent $>50 \%$ ). The distinction between limited and extensive disease was strongly associated with mortality (OR 5.24, 95\% CI 2.69-10.22; $\mathrm{p}<0.001$ ) and was robust with adjustment for age, sex and comorbidity score (supplementary table S5).

TABLE 4 Unadjusted associations between mortality and core clinical and imaging variables in patients with chest radiographic findings compatible with COVID-19 infection examined using logistic regression

\begin{tabular}{lcc} 
& OR $(95 \% \mathrm{CI})$ for mortality & p-value \\
\hline Age (years) & $1.07(1.04-1.10)$ & $<0.001$ \\
Sex & $0.82(0.44-1.53)$ & 0.53 \\
Duration of dyspnoea (days) & $1.01(0.92-1.11)$ & 0.78 \\
Duration of cough (days) & $0.91(0.83-0.99)$ & 0.03 \\
Duration of fever (days) & $0.85(0.78-0.93)$ & $<0.001$ \\
Duration of symptoms (days) & $0.86(0.78-0.93)$ & $<0.001$ \\
\hline Comorbidity score (0-4) & $1.52(1.12-2.05)$ & $<0.01$ \\
Chest radiography severity (1-3) & $2.38(1.61-3.50)$ & $<0.001$ \\
CT severity (1-3) & $2.62(1.67-4.10)$ & $<0.001$ \\
\hline Imaging (chest radiography/CT) severity (0 or 1) & $5.24(2.69-10.22)$ & $<0.001$ \\
\hline CT: computed tomography. & & \\
\hline
\end{tabular}


Prognostic scoring systems using reconstructed chest radiography and HRCT

For each of the three aforementioned models, AUC values fell minimally when multivariable analysis was confined to age and extent of disease on imaging (supplementary table S5). Therefore, simplified scoring systems for reconstructed chest radiography and HRCT were constructed based on these two variables. Age was categorised as $<60$ (score 0), 60-74 (score 1) and $>74$ (score 2) years, dividing the cohort into approximate thirds (OR 2.79, 95\% CI 1.80-4.31; p<0.001). Imaging extent scores were categorised as $<20 \%$ (score 0 ), $20-50 \%$ (score 1) and $>50 \%$ (score 2) (figures 2-4). Age and imaging scores were summed to provide five-point scales (0-4) for age/reconstructed chest radiography and age/HRCT scoring systems. Mortality in relation to the age/reconstructed chest radiography and age/HRCT scores is shown in figure 5 and supplementary table S6.

In a logistic regression model, the age/reconstructed chest radiography score was associated with mortality (OR 2.85, 95\% CI 2.00-4.06; $\mathrm{p}<0.001$ ) (AUC 0.77). This finding was reproduced in the validation cohort (OR 2.48, 95\% CI 1.47-4.17; $\mathrm{p}<0.001$ ) (AUC 0.73). In a separate logistic regression model, the age/ HRCT score was associated with mortality (OR 2.71, 95\% CI 1.92-3.84; p<0.005) (AUC 0.76). This finding was reproduced in the validation cohort (OR 4.23, 95\% CI 2.11-8.48; p<0.001) (AUC 0.80).

\section{Discussion}

In this simulated COVID-19 pandemic triage for a high influx of patients with mostly moderate-to-severe clinical features suspicious of COVID-19 pneumonia, the use of reconstructed chest radiography to determine morphology and extent of pneumonia allowed fair interobserver agreement for clinical management; however, reconstructed chest radiography with moderate disease extent displayed limited yield that could be assisted by integration with HRCT. The interobserver agreement considerably increased when using HRCT, which led to an increase in the number of recommended hospitalisations. This observation is of particular relevance as the observers were from different countries, used various COVID-19 triage strategies in their daily practice and had different subspecialty interests.

The major source of disagreement for the reconstructed chest radiography-based protocol was the frequency of requested HRCT scans, which ranged from $8 \%$ to $46 \%$ of cases. When HRCT work-up was not recommended by any of the study observers, the agreement was similar to that obtained with the HRCT-based protocol. Although we observed a tendency for considering the reconstructed chest radiography sufficient in patients with milder pulmonary dysfunction, abnormal reconstructed chest radiography and high exposure risk, the variety in requesting HRCT scans still had no obvious reasons (e.g. age, disease extent on reconstructed chest radiography, etc.). Such observation emphasises the need to define the clinical indications for CT scanning when the triage workflow relies on chest radiography as the first imaging modality. In fact, poorly defined criteria may unreasonably increase the number of CT scans after chest radiography, with potential detrimental effects on workflow and healthcare worker safety.

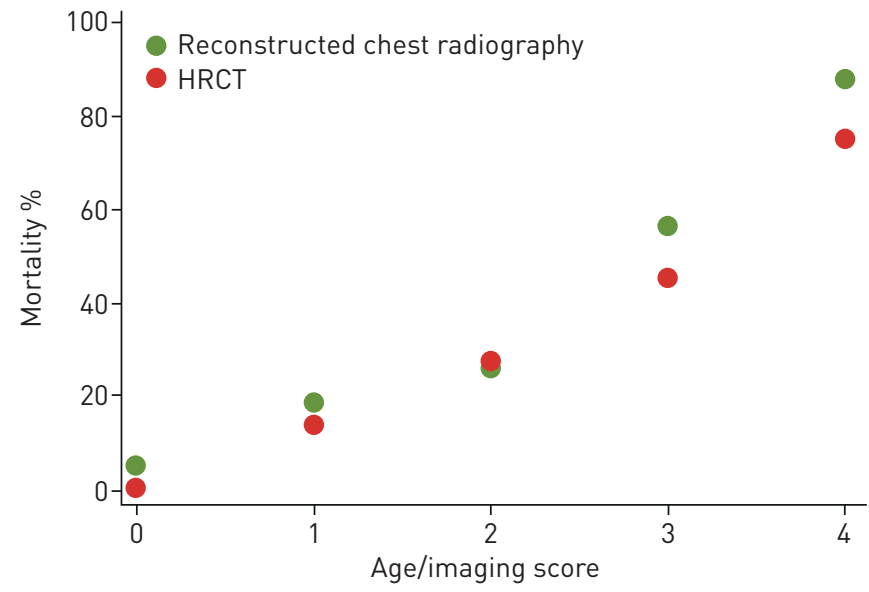

FIGURE 5 Mortality in relation to the age/reconstructed chest radiography and age/high-resolution computed tomography (HRCT) scores. Age and extent of disease on imaging scores were summed to provide five-point scales (0-4) for both the age/reconstructed chest radiography and age/HRCT scoring systems (see main text for details). 
In order to evaluate the clinical implications of the two imaging modalities, the sensitivity, specificity and prognostic value of reconstructed chest radiography and HRCT were compared. The levels of sensitivity for reconstructed chest radiography were in keeping with previous findings [9, 16, 17, 19]. The sensitivity, specificity and PPV of reconstructed chest radiography were marginally lower than HRCT, whereas a major discrepancy was found for the NPV due to the remarkable proportion of false-negative reconstructed chest radiographs.

Any optimisation process of COVID-19 triage protocols should consider mortality data and resource utilisation predictions [20]. First, the proportion of deaths among patients with normal reconstructed chest radiography was higher (12\%) compared with normal HRCT (5\%). Such a discrepancy was also evident by comparing reconstructed chest radiography- and HRCT-based prognostic scoring systems (figure 5). Moreover, the distinction between typical COVID-19 pneumonia and indeterminate findings was not worthwhile for both reconstructed chest radiography and HRCT as they were associated with similar outcomes. This suggests that, in a pandemic situation, indeterminate chest radiography or CT findings likely represent signs of COVID-19 pneumonia. Hence, after grouping indeterminate and typical COVID-19 categories, we found that the diagnostic categories had fairly major prognostic significance for both reconstructed chest radiography and HRCT. This finding is important as the diagnostic categories used in the present study substantially overlap both CO-RADS (COVID-19 Reporting and Data System) categories and those proposed by the Radiological Society of North America [8, 21].

Our findings do not suggest that HRCT extent scoring adds greatly to prognostic evaluation, over and above chest radiography scoring, and this is concordant with the need for a first-line approach by chest radiography to optimise use of radiology resources and maximise patient and healthcare employee safety. However, an alternative strategy, i.e. HRCT complement when chest radiography extent findings are intermediate, allowed categorisation into limited and extensive disease with a major prognostic separation. The proposed contingent staging algorithm, where reconstructed chest radiography grades $1(<20 \%)$ and 3 $(>50 \%)$ are accepted and HRCT is used to adjudicate on reconstructed chest radiography grade 2 (21$50 \%$ ), showed the highest prognostic value. Separation into a higher/lower risk dichotomy may have major clinical value, but we suggest that this observation requires further exploration if CT is to be integrated into routine prognostic evaluation in selected cases, when chest radiography findings are not definitive.

The higher number of deaths in subjects with normal reconstructed chest radiography still represents the main limitation of this staging system. In patients dying despite a reconstructed chest radiography grade of 1 , the HRCT extent grade was either intermediate or extensive in two-thirds of cases. These data suggest that a first-line approach by chest radiography will require some improvement in terms of sensitivity. Otherwise, a first-line approach by HRCT would provide major confidence in clinical decision and prognostication, and therefore it might considered in the context of local logistics that minimise risk of contamination (e.g. a dedicated scanner in the proximity of the triage rooms).

This study has some limitations. It is worth emphasising that our observations were derived from a pre-peak endemic environment (e.g. when RT-PCR demand was beyond capacity), while the triage process is being adjusted as the endemic changes. Nevertheless, the study findings may still be helpful in epidemic scenarios; the increased availability of the laboratory test results within a few hours could further mitigate the chest radiography limitations (e.g. lower diagnostic accuracy compared with CT), encouraging its use. Moreover, the reported heterogeneity in the requests for HRCT scans among the study observers when first interpreting a chest radiography might be overrated as a simulated triage setting does not likely reflect the routine clinical context, where multidisciplinary discussion (e.g. between radiologists and triagers) could support other clinical decisions with better integration of available data. The chest radiography was derived from HRCT data given the impossibility of running a two-arm randomised controlled trial between chest radiography and CT in the COVID-19 setting, yet we tested this approach against original chest radiography (supplementary material).

In conclusion, this study showed that reconstructed chest radiography findings were often regarded as not sufficiently informative by clinicians in a COVID-19 pandemic triage setting. This observation suggests that chest CT should be considered after chest radiography in a substantial percentage of patients with suspected COVID-19 pneumonia, thus potentially causing detrimental effects in the absence of predefined diagnostic work-up criteria. Nevertheless, the present study findings suggest that clinicians could rely on positive chest radiography showing a low or high extent of pneumonia, whereas an intermediate extent by chest radiography should be complemented by CT for optimal classification into high- and low-risk groups.

Data sharing: Data collected for the study will not be made available to others. 
Conflict of interests: N. Sverzellati has nothing to disclose. C.J. Ryerson has nothing to disclose. G. Milanese has nothing to disclose. E.A. Renzoni has nothing to disclose. A. Volpi has nothing to disclose. P. Spagnolo reports grants, personal fees and nonfinancial support from Roche, PPM Services and Boehringer Ingelheim, personal fees from Red X Pharma, Galapagos and Chiesi, outside the submitted work; and the author's spouse is an employee of Novartis. F. Bonella reports personal fees and nonfinancial support from Boehringer Ingelheim, Roche, Galapagos, Savara Pharma and Bristol Myers Squibb, outside the submitted work. I. Comelli has nothing to disclose. P. Affanni has nothing to disclose. L. Veronesi has nothing to disclose. C. Manna has nothing to disclose. A. Ciuni has nothing to disclose. C. Sartorio has nothing to disclose. G. Tringali has nothing to disclose. M. Silva has nothing to disclose. E. Michieletti has nothing to disclose. D. Colombi has nothing to disclose. A.U. Wells reports personal fees and other from Roche, Boehringer Ingelheim and Bayer, outside the submitted work.

\section{References}

1 Poston JT, Patel BK, Davis AM. Management of critically ill adults with COVID-19. JAMA 2020; 323: 1839-1841.

2 World Health Organization. Director-General's opening remarks at the media briefing on COVID-19 - 29 June 2020. 2020. www.who.int/director-general/speeches/detail/who-director-general-s-opening-remarks-at-themedia-briefing-on-covid-19---29-june-2020 Date last accessed: 5 February 2020.

3 Lancet Infectious Diseases. Challenges of coronavirus disease 2019. Lancet Infect Dis 2020; 20: 261.

4 Blažić I, Brkljačić B, Frija G. The use of imaging in COVID-19 - results of a global survey by the International Society of Radiology. Eur Radiol 2021; 31: 1185-1193.

5 American College of Radiology. ACR Recommendations for the use of Chest Radiography and Computed Tomography (CT) for Suspected COVID-19 Infection. 2020. www.acr.org/Advocacy-and-Economics/ ACR-Position-Statements/Recommendations-for-Chest-Radiography-and-CT-for-Suspected-COVID19-Infection Date last accessed: 5 February 2020.

$6 \mathrm{Xu}$ Q, Xu K, Yang C, et al. Askin tumor: four case reports and a review of the literature. Cancer Imaging 2011; 11: $184-188$.

7 Rubin GD, Ryerson CJ, Haramati LB, et al. The role of chest imaging in patient management during the COVID-19 pandemic: a multinational consensus statement from the Fleischner Society. Chest 2020; 158: 106-116.

8 Sverzellati N, Milanese G, Milone F, et al. Integrated radiologic algorithm for COVID-19 pandemic. J Thorac Imaging 2020; 35: 228-233.

9 Ai T, Yang Z, Hou H, et al. Correlation of chest CT and RT-PCR testing for coronavirus disease 2019 (COVID-19) in China: a report of 1014 cases. Radiology 2020; 296: E32-E40.

10 Fang Y, Zhang H, Xie J, et al. Sensitivity of chest CT for COVID-19: comparison to RT-PCR. Radiology 2020; 296: E115-E117.

11 Wang YXJ, Liu WH, Yang M, et al. The role of CT for Covid-19 patient's management remains poorly defined. Ann Transl Med 2020; 8: 145.

12 Kooraki S, Hosseiny M, Myers L, et al. Coronavirus (COVID-19) outbreak: what the department of radiology should know. J Am Coll Radiol 2020; 17: 447-451.

13 Davarpanah AH, Mahdavi A, Sabri A, et al. Novel screening and triage strategy in Iran during deadly coronavirus disease 2019 (COVID-19) epidemic: value of humanitarian teleconsultation service. J Am Coll Radiol 2020; 17: 734-738.

14 Mossa-Basha M, Medverd J, Linnau K, et al. Policies and guidelines for COVID-19 preparedness: experiences from the University of Washington. Radiology 2020; 296: E26-E31.

15 Oudkerk M, Buller HR, Kuijpers D, et al. Diagnosis, prevention, and treatment of thromboembolic complications in COVID-19: report of the National Institute for Public Health of the Netherlands. Radiology 2020; 297: E216-E222.

16 Schiaffino S, Tritella S, Cozzi A, et al. Diagnostic performance of chest X-ray for COVID-19 pneumonia during the SARS-CoV-2 pandemic in Lombardy, Italy. J Thorac Imaging 2020; 35: W105-W106.

17 Balbi M, Caroli A, Corsi A, et al. Chest X-ray for predicting mortality and the need for ventilatory support in COVID-19 patients presenting to the emergency department. Eur Radiol 2021; 31: 1999-2012.

18 Lee KH, Kim YH, Hahn S, et al. Computed tomography diagnosis of acute appendicitis: advantages of reviewing thin-section datasets using sliding slab average intensity projection technique. Invest Radiol 2006; 41: 579-585.

19 Wong HYF, Lam HYS, Fong AH, et al. Frequency and distribution of chest radiographic findings in patients positive for COVID-19. Radiology 2020; 296: E72-E78.

20 Maves RC, Downar J, Dichter JR, et al. Triage of scarce critical care resources in COVID-19 an implementation guide for regional allocation: an expert panel report of the task force for mass critical care and the American College of Chest Physicians. Chest 2020; 158: 212-225.

21 Simpson S, Kay FU, Abbara S, et al. Radiological Society of North America Expert Consensus Statement on Reporting Chest CT Findings Related to COVID-19. Endorsed by the Society of Thoracic Radiology, the American College of Radiology, and RSNA - Secondary Publication. J Thorac Imaging 2020; 35: 219-227. 